\title{
Association of Longitudinal Change in High-Sensitivity Troponin with All-Cause Mortality in Coronary Artery Disease: The Heart and Soul Study
}

\author{
Yaanik B. Desai $^{a}$ Rakesh K. Mishra ${ }^{b}$ Qizhi Fang ${ }^{b}$ Mary A. Whooleya, c \\ Nelson B. Schiller ${ }^{\text {b }}$ \\ a Department of Medicine, UCSF School of Medicine, San Francisco, CA, USA; \\ ${ }^{b}$ Division of Cardiology, UCSF School of Medicine, San Francisco, CA, USA; \\ 'Section of General Internal Medicine, Veterans Affairs Medical Center, San Francisco, CA, USA
}

\section{Keywords}

Coronary artery disease $\cdot$ Troponin $\cdot$ Biomarkers .

Cardiovascular disease risk factors

\begin{abstract}
Background: Serial increases in high-sensitivity cardiac troponin (hs-cTnT) have been associated with death in community-dwelling adults, but the association remains uninvestigated in those with coronary artery disease (CAD). Methods: We measured hs-cTnT at baseline and after 5 years in 635 ambulatory Heart and Soul Study patients with CAD. We also performed echocardiography at rest and after treadmill exercise at baseline and after 5 years. Participants were subsequently followed for the outcome of death. We used a multivariable-adjusted Cox proportional hazards model to evaluate the association between 5-year change in hs-cTnT and subsequent all-cause mortality. Results: Of the 635 subjects, there were 386 participants (61\%) who had an increase in hscTnT levels between baseline and year 5 measurements (median increase $5.6 \mathrm{pg} / \mathrm{mL}, \mathrm{IQR} 3.2-9.9 \mathrm{pg} / \mathrm{mL}$ ). There were 182 deaths after a mean 4.2-year follow-up after the year 5 visit. After adjusting for clinical variables, a $>50 \%$ increase in hscTnT between baseline and year 5 was associated with a
\end{abstract}

\section{KARGER}

(c) 2020 S. Karger AG, Basel

E-Mail karger@karger.com

www.karger.com/crd nearly 2-fold increased risk of death from any cause (hazard ratio $1.7,95 \%$ confidence interval 1.1-2.7). When addition of year 5 hs-cTnT was compared to a model including clinical variables and baseline hs-cTnT, there was a modest but statistically significant increase in C-statistic from 0.82 to 0.83 $(p=0.04)$. Conclusion: In ambulatory patients with CAD, serial increases in hs-cTnT over time are associated with an increased risk of death.

(c) 2020 S. Karger AG, Basel

\section{Introduction}

The development of a high-sensitivity serum assay to detect cardiac troponin $\mathrm{T}$ ( $\mathrm{cTn} \mathrm{T}$ ) has revealed the presence of low circulating TnT levels in apparently healthy adults [1]. These data have elicited considerable interest in the clinical significance of these circulating levels and have sparked investigation into the potential use of highsensitivity cTnT (hs-cTnT) in screening and risk stratification of heart disease [2]. There is also potential utility

Yaanik B. Desai, MD

Department of Medicine, UCSF School of Medicine

505 Parnassus Avenue

San Francisco, CA 94143 (USA)

E-Mail yaanik.desai@ucsf.edu

Nelson B. Schiller, MD

Division of Cardiology, UCSF School of Medicine

535 Mission Bay Boulevard South

San Francisco, CA 94158 (USA)

E-Mail Nelson.Schiller@ucsf.edu 
of hs-cTnT as a biomarker for predicting cardiovascular events and overall mortality because hs-cTnT has been associated with increased risks of cardiovascular death and all-cause mortality in asymptomatic adults both with and without evidence of prior heart disease [3-5]. We have previously shown in a cohort of patients with stable coronary artery disease (CAD) that for every doubling of a single, baseline hs-cTnT measurement, there was a 1.4fold increase in risk of cardiovascular death [4]. Changes in hs-cTnT over time have been shown to add additional prognostic value compared with static measurements in community-dwelling adults [6-8], but it is unknown whether changes over time are prognostically meaningful in patients with known, stable CAD. Thus, in this study, we examine whether changes in hs-cTnT over 5 years are associated with an increased risk of all-cause mortality in a cohort of ambulatory patients with known CAD.

\section{Methods}

The Heart and Soul Cohort was originally designed to analyze the impact of depression on outcomes in patients with stable CAD. Details regarding recruitment of the cohort have been published previously $[4,9]$. As described, patients were included if they had evidence of stable CAD which was defined by: a prior history of myocardial infarction (MI), angiographic evidence of at least 50\% stenosis in 1 coronary vessel or more, evidence of inducible ischemia by treadmill echocardiography or nuclear perfusion stress imaging, or, finally, a previous history of a coronary revascularization procedure. Patients were excluded if they were unable to walk 1 block, if they had an MI within 6 months prior to enrollment, or if they planned to leave the Bay Area within 3 years after enrollment.

Between September 2000 and December 2002, participants $(n=1,024)$ from 12 outpatient clinics in the Bay Area were recruited. They completed baseline questionnaires to determine past medical history, and they had 12-h fasting morning blood samples drawn before completing baseline rest and stress echocardiograms. Blood samples were centrifuged and frozen at $-80^{\circ} \mathrm{C}$.

Of patients who completed the baseline examination, 984 had blood samples available for analysis. Of those participants, 745 survived to the year 5 examination. Of surviving participants, 635 (85\%) returned for a follow-up examination 5 years after enrollment. At the 5-year follow-up visit, participants again completed questionnaires and an exercise treadmill test with resting and stress echocardiograms. Twelve-hour fasting serum samples were once again obtained prior to stress echocardiography and frozen at $-80^{\circ} \mathrm{C}$.

Concentrations of hs-cTnT were measured with highly sensitive reagents on a commercially available analyzer (Elecsys 2010; Roche Diagnostics) for baseline and year 5 blood samples. The analytical measurement range of the assay was $5-10,000 \mathrm{pg} / \mathrm{mL}$. We defined hs-cTnT levels $<5 \mathrm{pg} / \mathrm{mL}$, which was the lower limit of detection on our analyzer, as "undetectable." We defined levels of $5-14 \mathrm{pg} / \mathrm{mL}$ as "detectable" and levels $>14 \mathrm{pg} / \mathrm{mL}$ as "elevated," which represents the 99th percentile in validation trials [10].
Demographic variables and medical history were assessed by questionnaires. Total and HDL cholesterol were measured on fasting serum samples, and estimated glomerular filtration rate (eGFR) was calculated using creatinine and cystatin $\mathrm{C}$ levels, which were also assessed based on fasting serum samples [11].

As previously described, patients underwent exercise stress testing according to a standard Bruce protocol with continuous EKG monitoring. Patients who were unable to complete the standard protocol were converted to a manual protocol. Prior to the exercise stress test, patients had a complete 2D echocardiogram with standard views using an Acuson Sequoia ultrasound system (Siemens Medical Solutions, Mountain View, CA, USA) with a 3.5-MHz transducer and Doppler ultrasound examination. Standard 2D parasternal short-axis, apical 2-chamber, apical 4-chamber, and subcostal views were obtained and used to measure chamber size and left ventricular (LV) ejection fraction (EF). The truncated ellipsoid method was used to estimate LV mass, which was indexed to body surface area [12]. Mitral inflow E- and A-wave velocities, E-wave deceleration time, and the ratio of the pulmonary venous systolic-to-diastolic flow velocity-time integral were used to assess LV diastolic dysfunction. Inducible ischemia was defined by the presence of new wall motion abnormality after peak exercise, using the wall motion score index (WMSI). The WMSI is a way to quantify the average degree of wall motion abnormality present across segments of the heart; a different score is assigned for normal $($ WMSI $=1)$, hypokinetic $($ WMSI $=2)$, akinetic $($ WMSI $=$ 3 ), and dyskinetic (WMSI $=4$ ) segments of the heart in various views, and a composite score is derived from the average score across segments [13]. Exercise capacity was measured as the total metabolic equivalents achieved during peak exercise. Echocardiograms were all read by a single, expert cardiologist (N.B.S.).

The primary outcome in this study was time to death from any cause. Death was verified by death certificates and review of medical records. As described previously in further detail, all events were adjudicated by independent and blinded reviewers [4].

To compare differences in year 5 characteristics among categories of year $5 \mathrm{hs}-\mathrm{cTnT}$ (i.e., undetectable, detectable, and elevated), we used the $\chi^{2}$ test for categorical variables and one-way analysis of variance (ANOVA) for continuous variables. We used an unadjusted and a multivariable-adjusted Cox proportional hazards model to evaluate the association between natural log-transformed 5 -year change in hs-cTnT and all-cause mortality. When evaluating relative change, we imputed a value of $3 \mathrm{pg} / \mathrm{mL}$ for all patients who had undetectable hs-cTnT values. Models were adjusted for demographic variables at year 5 (age, sex, race, smoking, and body mass index), clinical variables at year 5 (history of hypertension, diabetes mellitus, MI, congestive heart failure, coronary revascularization, total cholesterol, HDL cholesterol, and eGFR), and echocardiographic variables at year 5 (LVEF, LV mass index, diastolic dysfunction, exercise capacity, and inducible ischemia defined by WMSI after exercise). Diastolic dysfunction was defined as pseudonormal or restrictive filling on mitral inflow [14].

We also used a logistic regression model for all-cause mortality, adjusted for the clinical and demographic variables listed above, to calculate changes in the area under the receiver-operating curves (i.e. Harrell's C-statistic) with addition of log-transformed hscTnT. C-statistics for competing models were compared using the DeLong test $[15,16]$. We evaluated calibration of the logistic regression model with the Hosmer-Lemeshow goodness-of fit-test with 10 groups. 
We also calculated the category-free net reclassification index (NRI), which estimates how well a new biomarker "reclassifies" patients to the correct outcome (in our case death or no death). The logistic regression model was used to calculate the probability of all-cause mortality $\left(P_{0}\right)$. This probability was then recalculated for the outcome of death with the addition of baseline hs-cTnT, year 5 hs-cTnT, or change in hs-cTnT levels $\left(P_{x, y \text {, or } z}\right)$. In each test, if $P_{x, y, \text { or } z}>\mathrm{P}_{0}$, then the person had an upward reclassification, and if $P_{x, y, \text { or } z}<P_{0}$, then the person had a downward reclassification. The NRI represents the sum of the proportion of patients who are correctly "upclassified" to the outcome of death and the proportion of patients who are correctly "downclassified" to the nonoutcome $[17,18]$. We also calculated the integrated discrimination improvement (IDI) statistic, which represents the improvement in the average predicted risk for death. The IDI is calculated using the formula IDI $=\left(P_{a}-P_{b}\right)-\left(P_{x}-P_{y}\right)$, where $P_{a}$ and $P_{x}$ are the mean probabilities of having the outcome of interest (in our case death) with and without incorporation of the additional biomarker (in our case, baseline, year 5, or change in hs-cTnT) respectively, and $P_{b}$ and $\mathrm{P}_{y}$ represent the mean probability of not having the outcome of interest with and without incorporation of the additional biomarker, respectively $[17,18]$.

Analyses were done using STATA (version 13; StataCorp) and $\mathrm{R}$ (version 3.5.0). For the AUC analyses, we used the pROC package for R [19], and for NRI and IDI analyses we used the PredictABEL package [20].

\section{Results}

In the cohort of 635 patients, 225 had undetectable hscTnT at year 5, 216 had a detectable level, and 194 had an elevated level. There were 386 participants (61\%) who had an increase in hs-cTnT levels between baseline and year 5 measurements (median increase $5.6 \mathrm{pg} / \mathrm{mL}$, IQR 3.2-9.9 pg/mL). Patients with higher hs-cTnT at year 5 were more likely to be older, male, have diabetes mellitus, have congestive heart failure, and have lower eGFR (Table 1). They were also more likely to have had a coronary revascularization procedure and have larger LV mass index, poorer LV systolic and diastolic function, and inducible ischemia at year 5. There were 182 deaths after a mean 4.2-year follow-up after year 5. Of the 194 patients with elevated year 5 troponin, there were 103 deaths (53\%) during follow-up after year 5 .

There were 235 patients who had a $>50 \%$ increase in hscTnT between baseline and year 5 , which was associated with a significantly higher risk of all-cause death in the model fully adjusted for demographic, clinical, and echocardiographic variables (HR 1.7, 95\% CI 1.1-2.7) (Table 2). A $>25 \%$ increase in hs-cTnT was not associated with a significant change in mortality in fully adjusted models.

There were 19 patients who had a $>50 \%$ decrease in hs-cTnT, but this was not associated with a significant change in mortality in fully adjusted models. Absolute change in hs-cTnT was also not significantly associated with mortality in the fully adjusted Cox regression model (HR 0.9, 95\% CI 0.5-5).

In the fully adjusted model, the risk of death was not significantly increased for the 112 patients who went from undetectable baseline (i.e., $<5 \mathrm{pg} / \mathrm{mL}$ ) to year $5 \mathrm{lev}$ els $>5 \mathrm{pg} / \mathrm{mL}$ (HR 0.5, 95\% CI 0.2-2.1), nor was there an increased risk associated with going from nonelevated baseline levels (i.e., $<14 \mathrm{pg} / \mathrm{mL}$ ) to elevated year 5 levels (i.e., $>14 \mathrm{pg} / \mathrm{mL}$ ) (HR 1.7, 95\% CI 0.9-3.1).

In the model adjusted for demographic and clinical variables, patients who had undetectable baseline hscTnT but elevated level at year 5 had a 3 -fold increased risk of death (HR 3.1, 95\% CI 1.0-9.9) compared to patients who had undetectable levels at both baseline and year 5 (Table 3; Fig. 1a). Patients who had detectable hscTnT level at baseline and either detectable or elevated level at year 5 also had an increased risk of death in this model (HR 2.3, 95\% CI 1.2-4.5, and HR 3.5, 95\% CI 1.77.0 , respectively), as did patients who had elevated baseline levels and either detectable or elevated year 5 level (HR 5.6, 95\% CI 2.3-13.6, and HR 3.5, 95\% CI 1.8-6.9, respectively).

In the model fully adjusted for demographic, clinical, and also echocardiographic variables, patients who had baseline detectable levels of hs-cTnT but elevated levels at year 5 had a nearly 3 -fold increased risk of death (HR 2.7, 95\% CI 1.1-6.6) (Table 3; Fig. 1b). The 12 patients who went from elevated baseline levels to detectable year 5 levels had a 4-fold increased risk of death (HR 4.1, 95\% CI 1.3-13.0). The 12 patients who had undetectable baseline levels but elevated year 5 levels did not have a significantly different risk of death (HR 3.1, 95\% CI 0.7-12.5). The increase from the undetectable baseline level to the detectable year 5 level was not associated with an increased risk of death (HR 1.5, 95\% CI 0.6-3.8). Patients who had detectable levels at baseline and year 5 also did not have a significant change in risk of death (HR 1.9, 95\% CI 0.84.2). Patients who had elevated levels at baseline and at year 5 also did not have a significant change in risk of death (HR 2.0, 95\% CI 0.8-5.0). There were too few patients who went from having detectable or elevated baseline levels to undetectable year 5 levels, and given incomplete data on these patients, we were unable to evaluate these HRs for death with full adjustment.

When compared to a model of clinical variables, the addition of baseline hs-cTnT did not significantly improve model discrimination measured by C-statistic; however, the addition of year 5 hs-cTnT did increase C-statistic 
Table 1. Patient characteristics stratified by hs-cTnT at year 5

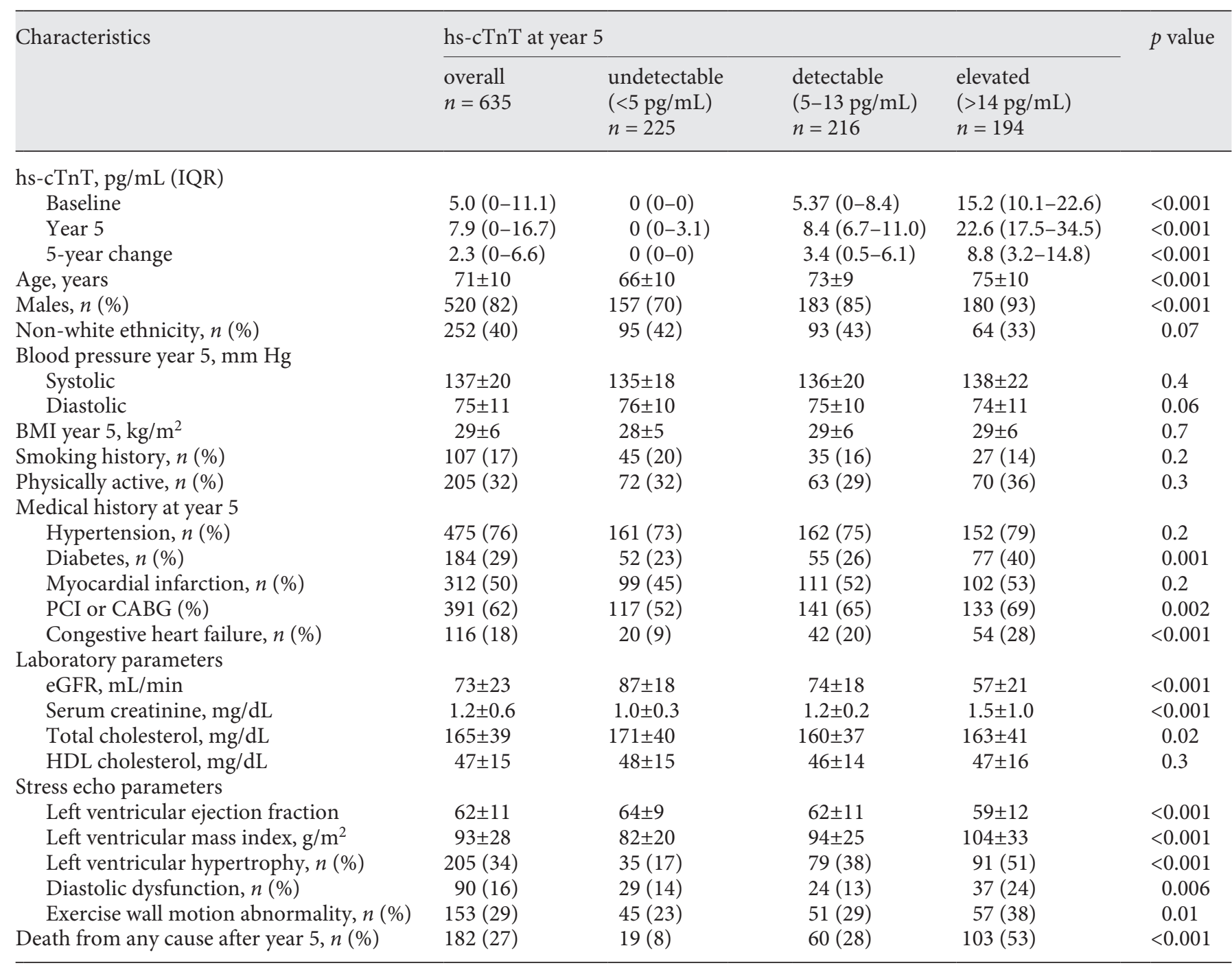

Table 2. Risk of death based on relative change in hs-cTnT levels between baseline and year 5

\begin{tabular}{|c|c|c|c|}
\hline Death of any cause, $n(\%)$ & $8(42)$ & $83(22)$ & $91(39)$ \\
\hline HR unadjusted model & $1.1(0.5-2.4)$ & 1.0 & $2.0(1.5-2.7)$ \\
\hline HR adjusted for demographic variables (95\% CI) & $0.4(0.1-1.6)$ & 1.0 & $1.9(1.4-2.6)$ \\
\hline HR adjusted for demographic + clinical variables $(95 \% \mathrm{CI})$ & $0.6(0.2-1.6)$ & 1.0 & $1.8(1.3-2.5)$ \\
\hline
\end{tabular}

Demographic variables were: age, sex, race, smoking, and BMI. Clinical variables were history of hypertension, diabetes, myocardial infarction, congestive heart failure, revascularization procedure, total and HDL cholesterol level, and estimated glomerular filtration rate. Echocardiographic variables were: left ventricular ejection fraction and mass index, diastolic dysfunction, exercise capacity, and presence of inducible ischemia. 


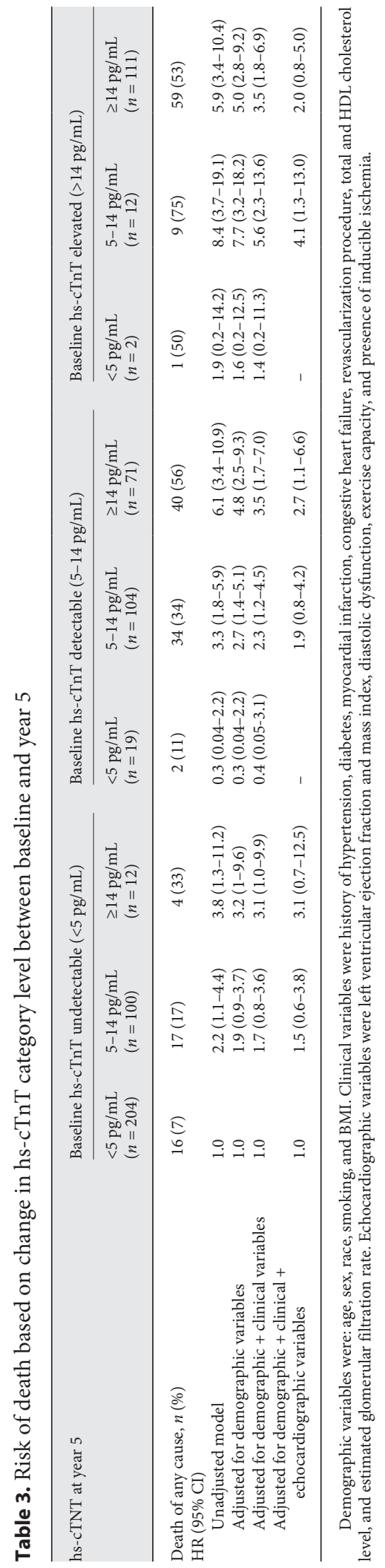

hs-cTnT and All-Cause Mortality in CAD from 0.80 to $0.83(p<0.01)$ (Table 4). When absolute change in hs-cTnT troponin was included in models, Cstatistic did not increase. However, when addition of year 5 hs-cTnT was compared to a model including clinical variables and baseline hs-cTnT, there was a modest but statistically significant increase in C-statistic from 0.82 to $0.83(p=0.04)$. Additionally, the category-free NRI in this model was 0.36 (95\% CI 0.18-0.53), meaning the sum of the proportion of patients who were either correctly upclassified to a higher probability of death in this model and patients who were correctly downclassified to having a lower probability of death was $36 \%$. The IDI statistic was also significantly increased (HR 0.015 ; 95\% CI $0.0043-$ 0.026 ) when the addition of year $5 \mathrm{hs}-\mathrm{cTnT}$ was compared to a clinical model with baseline hs-cTnT (Table 4).

\section{Discussion}

Our study shows that in a population of patients with stable CAD, increase in hs-cTnT over 5 years is associated with an increased risk of death - in particular, a 50\% relative increase is associated with a nearly 2 -fold increase in risk of death, and addition of year $5 \mathrm{hs}$-cTnT improves discrimination and reclassification compared to a model with clinical risk factors and baseline hs-cTnT. In a model adjusted for demographic and clinical variables alone, patients whose hs-cTnT level increased to a higher category by year 5 generally had a higher risk of death than patients whose hs-cTnT level was undetectable at baseline and year 5, as shown in the Figure 1a. In the full model, where we also adjusted for echocardiographic variables, an increase from detectable to elevated levels was associated with a nearly 3 -fold increased risk for death as shown in the Figure $1 \mathrm{~b}$, though this finding was not apparent across most of the subgroups, suggesting that hs-cTnT may be a marker for known echocardiographic variables rather than an independent predictor.

Patients with elevated baseline and year $5 \mathrm{hs-cTnT}$ did not have a significantly higher rate of death when echocardiographic variables were adjusted for in the model, though these patients did have an increased risk in the model adjusted only for demographic and clinical variables, which is consistent with prior studies. It should again be emphasized, however, that unlike previously published work on hs-cTnT changes, we were able to control for echocardiographic variables. Therefore, the loss of a statistically significant increase in the risk of death with adjustment for echocardiographic variables may suggest that in this population of patients with coronary 


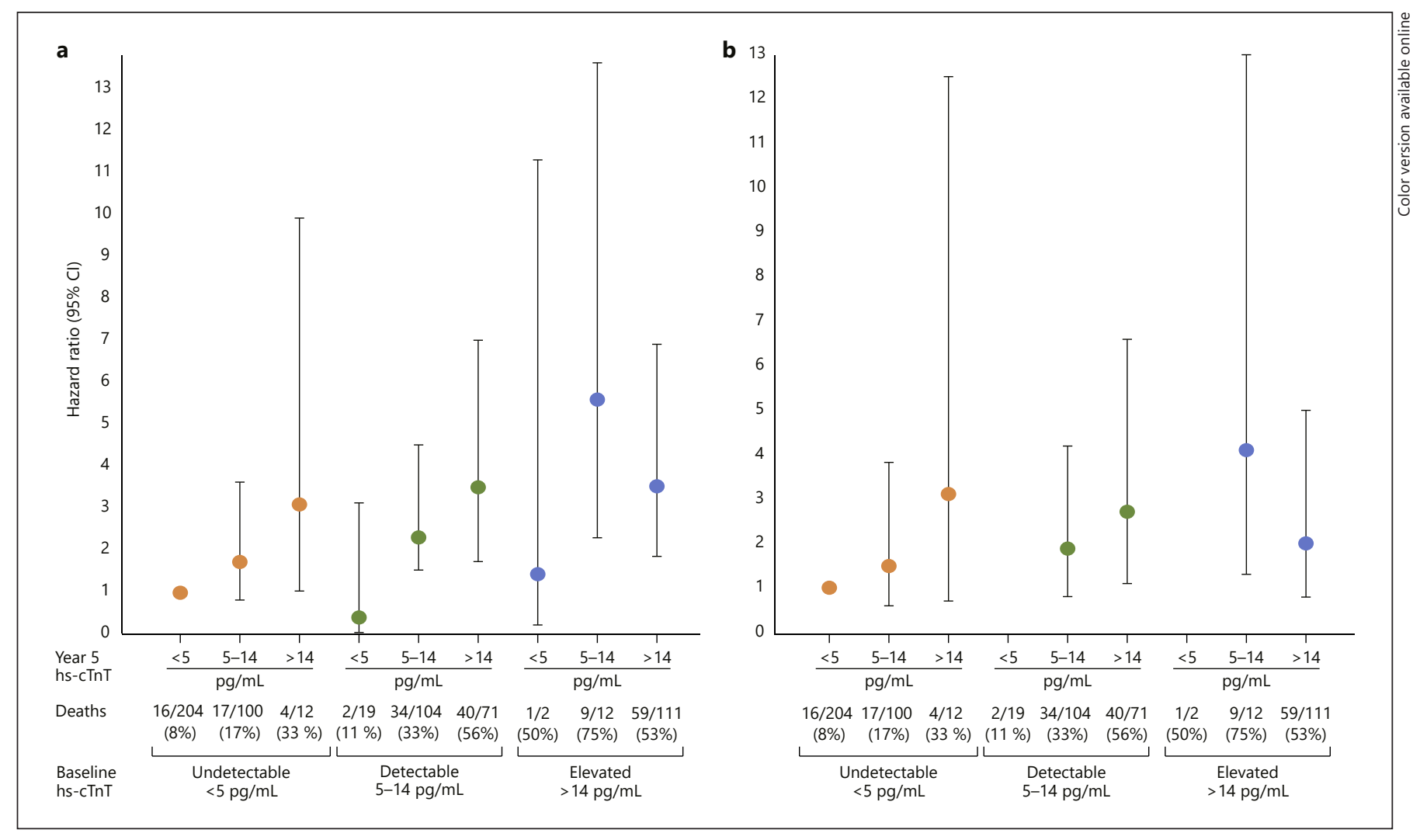

Fig. 1. Hazard ratios for death from any cause categorized by levels of baseline and year 5 high-sensitivity cardiac troponin (hs-cTnT). a Data adjusted for clinical and demographic variables. b Data adjusted for clinical, demographic, and echocardiographic variables. Error bars represent $95 \%$ confidence intervals. Data for patients who had detectable baseline but undetectable year 5 levels as well as patients with elevated baseline but undetectable year 5 levels are not displayed because we were not able to generate fully adjusted hazard ratios due to small numbers of deaths and missing data in these categories.

Table 4. Prediction of death using C-statistic, net reclassification index (NRI), and integrated discrimination improvement (IDI) in clinical models with addition of baseline and year 5 hs-cTnT levels

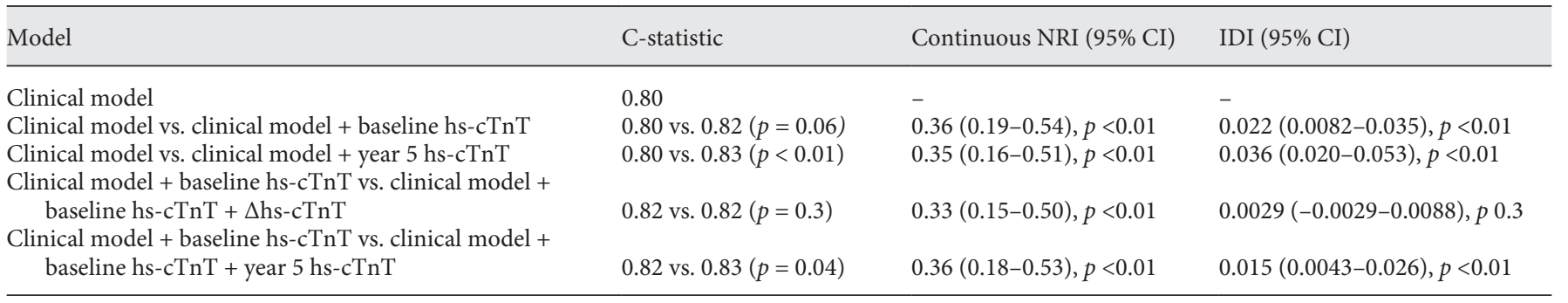

Clincal model includes age, sex, race, smoking status, BMI, year 5 eGFR, HDL, total cholesterol, and history of DM, HTN, CHF, MI, and CABG at year 5. $\Delta$ hs-cTnT reflects the absolute change from baseline to year $5 \mathrm{hs-cTnT}$. All values of hs-cTnT and $\Delta \mathrm{hs}$-c TnT were natural log transformed.

artery disease, stably elevated hs-cTnT over time could simply be a marker for echocardiographic variables such as LV mass index, low EF, and inducible ischemia, whereas in patients who increased from detectable to elevated levels, hs-cTnT was an independent marker for death.
The fact that hs-cTnT is a marker for echocardiographic parameters such as low EF, large LV mass index, or presence of inducible ischemia should not be surprising, as prior authors have hypothesized that levels of hscTnT in asymptomatic adults may reflect ongoing occult 
ischemia [8], and parameters such as large LV mass index and low EF are known independent predictors of mortality $[21,22]$; this finding would not detract from potential clinical applicability of the biomarker in risk prediction. It is interesting, however, that there was still a significant increase in risk of death in patients with a $>50 \%$ relative increase in hs-cTnT and in patients whose levels increased from detectable baseline to elevated year 5 levels, even after adjusting for echocardiographic variables. The pathophysiology underlying the low levels of troponin remains an area of study, but our finding that hs-cTnT predicts mortality independently of the presence of inducible ischemia and other echocardiographic variables may be consistent with additional, alternative proposed mechanisms of troponin release other than myocardial necrosis [23].

Another interesting finding is that the 100 patients who went from undetectable baseline to detectable year 5 hs-cTnT levels had no significant mortality increase, neither in the fully adjusted model nor in the model adjusted for demographic and clinical variables. This is in contrast to data presented by McEvoy et al. [7], who did show a mortality association in a similar group. This suggests that in our population of patients with $\mathrm{CAD}$, the association of change in hs-cTnT with increased risk of death is only present in patients with either higher baseline hscTnT level or with a larger magnitude of change in hscTnT.

We report changes in hs-cTnT serially measured over 5 years, but the optimal time interval over which to measure hs-cTnT for prognostication is unclear. McEvoy et al. [7] demonstrated the association with 6-year change and death, and Glick et al. [8] and DeFilippi et al. [6] showed an association with cardiovascular death over a 2 - to 3-year change [6-8]. The 1.7-fold increased risk of death associated with $>50 \%$ increase in hs-cTnT is comparable to the HR of 1.4 reported by McEvoy et al. [7] over a 6-year time period. Further study is required, perhaps with multiple, more frequent serial measurements, to determine the temporal dynamics of troponin change as it relates to cardiovascular outcome.

A major limitation of our study is the smaller size of our cohort in comparison to previous studies that have investigated change in hs-cTnT over time; this may have limited our power to detect a true difference in risk of mortality in certain subgroups and, alternatively, may have led to a statistically significant association due to chance (as may have been the case in the 12 patients who went from elevated baseline hs-cTnT to detectable levels at year 5 in the fully adjusted model).

hs-cTnT and All-Cause Mortality in CAD
Prior studies have shown that a decrease in hs-cTnT over time is associated with decreased mortality [7]. This was not demonstrated in our study. Our population of patients is unique in that they already have known CAD, which may explain why there were so few patients who had any decrease in hs-cTnT at all. There were only 19 patients in our cohort of 635 patients who had a $>50 \%$ decrease in hs-cTnT over the study period. Similarly, there were only 2 patients who went from elevated baseline (i.e., $>14 \mathrm{pg} / \mathrm{mL}$ ) to undetectable year 5 levels. Across the board, we had few patients with a decrease in hs-cTnT over time, and this may suggest that our study was underpowered to detect a difference in mortality with a decrease in hs-cTnT.

Additional limitations to our study include that it was a retrospective observational study, and while we attempted to control for possible confounders in our adjusted model, it is possible that change in hs-cTnT over time is a marker of additional known parameters that we did not control for in our study.

Another limitation that may have affected the interpretation of our results is that patients who died after the initial visit, but before the year 5 visit, were not included in this analysis because they passed away before change in hscTnT could be evaluated; this may have led to a "healthy survivor" bias because these patients were disproportionately more likely to have an initially elevated hs-cTnT level, meaning that the patients in the baseline "elevated" subgroup with initially highest risk of death had already died before we could evaluate the effect of the 5 -year change; this finding warrants further study with more frequent serial hscTnT troponin measurements to more accurately predict risk in this population. Our study also has a predominantly male population, which may limit generalizability.

In summary, we show that increase in hs-cTnT over 5 years is associated with an increased risk of death in patients with stable CAD, though further study in additional patients with more frequent serial measurements is needed to elucidate the prognostic role in this population.

\section{Acknowledgment}

We thank all members of Dr. Nelson B. Schiller's Physiology Lab and Research Group for their discussion of the findings during group meetings.

\section{Statement of Ethics}

The institutional review board at each of the sites approved this protocol. All participants provided written informed consent.

Cardiology 2020;145:63-70

DOI: $10.1159 / 000503954$ 


\section{Disclosure Statement}

The authors have no conflicts of interest to declare.

\section{Funding Sources}

This work was supported by the National Heart, Lung, and Blood Institute (R01 HL079235 to M.A.W.); the Department of Veterans Affairs (Epidemiology Merit Review Program); the Robert Wood Johnson Foundation (Generalist Physician Faculty Scholars Program); the American Federation for Aging Research (Paul Beeson Faculty Scholars in Aging Research Program); the Veterans Health Research Institute; and an investigator-initiated grant from Roche Diagnostics, which funded the hs-cTnT assay.
None of these funding sources had any role in the design and conduct of the study; collection, management, analysis, and interpretation of the data; and preparation, review, or approval of the manuscript.

\section{Author Contributions}

Dr. Rakesh K. Mishra, Dr. Nelson B. Schiller, and Dr. Mary A. Whooley conceived of the idea for the manuscript. Dr. Yaanik B. Desai and Dr. Qizhi Feng carried out the statistical analysis. All authors reviewed and discussed the results, and suggested additional analyses. Dr. Yaanik B. Desai took the lead in writing the manuscript. All authors reviewed and contributed to the final manuscript.

\section{References}

1 de Lemos JA, Drazner MH, Omland T, Ayers CR, Khera A, Rohatgi A, et al. Association of troponin $\mathrm{T}$ detected with a highly sensitive assay and cardiac structure and mortality risk in the general population. JAMA. 2010 Dec; 304(22):2503-12

2 Sherwood MW, Kristin Newby L. High-sensitivity troponin assays: evidence, indications, and reasonable use. J Am Heart Assoc. 2014 Jan;3(1):e000403.

3 Omland T, de Lemos JA, Sabatine MS, Christophi CA, Rice MM, Jablonski KA, et al.; Prevention of Events with Angiotensin Converting Enzyme Inhibition (PEACE) Trial Investigators. A sensitive cardiac troponin $\mathrm{T}$ assay in stable coronary artery disease. $\mathrm{N}$ Engl $\mathrm{J}$ Med. 2009 Dec;361(26):2538-47.

4 Beatty AL, Ku IA, Christenson RH, DeFilippi CR, Schiller NB, Whooley MA. High-sensitivity cardiac troponin $T$ levels and secondary events in outpatients with coronary heart disease from the Heart and Soul Study. JAMA Intern Med. 2013 May;173(9):763-9.

5 Biener M, Giannitsis E, Lamerz J, MuellerHennessen M, Vafaie M, Katus HA. Prognostic value of elevated high-sensitivity cardiac troponin $T$ levels in a low risk outpatient population with cardiovascular disease. Eur Heart J Acute Cardiovasc Care. 2016 Oct;5(6):409_ 18.

6 deFilippi CR, de Lemos JA, Christenson RH, Gottdiener JS, Kop WJ, Zhan M, et al. Association of serial measures of cardiac troponin $\mathrm{T}$ using a sensitive assay with incident heart failure and cardiovascular mortality in older adults. JAMA. 2010 Dec;304(22):2494-502.

7 McEvoy JW, Chen Y, Ndumele CE, Solomon SD, Nambi V, Ballantyne CM, et al. Six-year change in high-sensitivity cardiac troponin $\mathrm{T}$ and risk of subsequent coronary heart disease, heart failure, and death. JAMA Cardiol. 2016 Aug;1(5):519-28.
8 Glick D, deFilippi CR, Christenson R, Gottdiener JS, Seliger SL. Long-term trajectory of two unique cardiac biomarkers and subsequent left ventricular structural pathology and risk of incident heart failure in community-dwelling older adults at low baseline risk. JACC Heart Fail. 2013 Aug;1(4):353-60.

9 Whooley MA, de Jonge P, Vittinghoff E, Otte C, Moos R, Carney RM, et al. Depressive symptoms, health behaviors, and risk of cardiovascular events in patients with coronary heart disease. JAMA. 2008 Nov;300(20): 2379-88.

10 Giannitsis E, Kurz K, Hallermayer K, Jarausch J, Jaffe AS, Katus HA. Analytical validation of a high-sensitivity cardiac troponin $\mathrm{T}$ assay. Clin Chem. 2010 Feb;56(2):254-61.

11 Inker LA, Schmid CH, Tighiouart H, Eckfeldt JH, Feldman HI, Greene T, et al.; CKD-EPI Investigators. Estimating glomerular filtration rate from serum creatinine and cystatin C. N Engl J Med. 2012 Jul;367(1):20-9.

12 Schiller NB, Shah PM, Crawford M, DeMaria A, Devereux R, Feigenbaum H, et al. Recommendations for quantitation of the left ventricle by two-dimensional echocardiography. American Society of Echocardiography Committee on Standards, Subcommittee on Quantitation of Two-Dimensional Echocardiograms. J Am Soc Echocardiogr. 1989 Sep-Oct; 2(5):358-67.

13 Lang RM, Badano LP, Mor-Avi V, Afilalo J, Armstrong A, Ernande L, et al. Recommendations for cardiac chamber quantification by echocardiography in adults: an update from the American Society of Echocardiography and the European Association of Cardiovascular Imaging. J Am Soc Echocardiogr. 2015 Jan;28(1):1-39.e14

14 Redfield MM, Jacobsen SJ, Burnett JC Jr, Mahoney DW, Bailey KR, Rodeheffer RJ. Burden of systolic and diastolic ventricular dysfunction in the community: appreciating the scope of the heart failure epidemic. JAMA. 2003 Jan; 289(2):194-202.
15 Harrell FE Jr, Lee KL, Mark DB. Multivariable prognostic models: issues in developing models, evaluating assumptions and adequacy, and measuring and reducing errors. Stat Med. 1996 Feb;15(4):361-87.

16 DeLong ER, DeLong DM, Clarke-Pearson DL. Comparing the areas under two or more correlated receiver operating characteristic curves: a nonparametric approach. Biometrics. 1988 Sep;44(3):837-45.

17 Pencina MJ, D’Agostino RB Sr, D’Agostino RB Jr, Vasan RS. Evaluating the added predictive ability of a new marker: from area under the ROC curve to reclassification and beyond. Stat Med. 2008 Jan;27(2):157-72.

18 Pencina MJ, D’Agostino RB Sr, Steyerberg EW. Extensions of net reclassification improvement calculations to measure usefulness of new biomarkers. Stat Med. 2011 Jan;30(1): $11-21$.

19 Robin X, Turck N, Hainard A, Tiberti N, Lisacek F, Sanchez JC, et al. pROC: an opensource package for $\mathrm{R}$ and $\mathrm{S}+$ to analyze and compare ROC curves. BMC Bioinformatics. 2011 Mar;12(1):77.

20 Kundu S, Aulchenko YS, van Duijn CM, Janssens AC. PredictABEL: an R package for the assessment of risk prediction models. Eur J Epidemiol. 2011 Apr;26(4):261-4.

21 Curtis JP, Sokol SI, Wang Y, Rathore SS, Ko DT, Jadbabaie F, et al. The association of left ventricular ejection fraction, mortality, and cause of death in stable outpatients with heart failure. J Am Coll Cardiol. 2003 Aug;42(4): $736-42$.

22 Abdi-Ali A, Miller RJ, Southern D, Zhang M, Mikami Y, Knudtson M, et al. LV mass independently predicts mortality and need for future revascularization in patients undergoing diagnostic coronary angiography. JACC Cardiovasc Imaging. 2018 Mar;11(3):423-33.

23 White HD. Pathobiology of troponin elevations: do elevations occur with myocardial ischemia as well as necrosis? J Am Coll Cardiol. 2011 Jun;57(24):2406-8. 